\title{
Design and Implementation of Prosthetic Hand Control using Myoelectric Signal
}

\author{
Akif Rahmatillah*, Limpat Salamat", Soegianto Soelistiono ${ }^{\#}$ \\ ${ }^{\#}$ Biomedical Engineering Study Program, Universitas Airlangga, C-Campus Mulyorejo, Surabaya, 60115, Indonesia \\ E-mail: ${ }^{* 1}$ akif-r@fst.unair.ac.id
}

\begin{abstract}
Amputation is a medical procedure that is required to cut part of or all of the extremity, i.e. upper limbs or lower limbs. In the final phase of the procedure, patients have to adapt to their new condition including the use of prostheses. Nowadays, Prosthetic hand have had a lot of improvements that enable patients to do normal activities by exploiting their myoelectric signal. This study has a goal to produce prosthetic hand that can respond to patient generating myoelectric signal. Three muscle leads ( 2 on muscle flexor digitorum, 1 on muscle extensor digitorum) were processed by 3 channels surface electromyography (sEMG) that contain of instrument amplifier i.e. high-pass filter, rectifier, and notch filter. Myoelectric signal is processed to extraction feature and classified by artificial neural network (ANN) that had been offline-trained before and had 21 neurons input layer, 10 neurons hidden layer, and 3 neurons output layer to detect 3 hand movements, i.e. grasping, pinch, and open grasp. ANN and prosthetic hand control was embedded on Arduino Due microcontroller so that the system could be used in stand-alone and real time mode. The results of the testing from 4 research subjects shown that the hand prostheses system had success rate of $87 \%-91 \%$.
\end{abstract}

Keywords — prosthetic hand; electromyography; artificial neural network; myoelectric signal.

\section{INTRODUCTION}

Amputation is a medical procedure that can save patients, in spite of its negative effect. Such effect, for instance, patients are unable to use their limbs as before (loss of functionalities). These negative effects also include stress due to combination of factors such as surgical procedures, the pain, limited movements, and also concerns about their future [1]. Patients who undergone amputation procedures, thereafter enter a stage of rehabilitation for about six months [2]. At this stage, patients will be trained various things related to the condition of the stumps of their limbs. These include mobility training, muscle strengthening exercises, and how to care for surgical scarring. At this rehabilitation stage, patients are also trained using prostheses as artificial parts that replacing the amputated limbs. The prosthesis has the primary goal of restoring limb function and also used as cosmetics [3]. Today, prosthesis has undergone fast developments with advances in technology, especially for bionic hand prosthesis by the implementation of myoelectric control [4]. For example, arm prosthesis is one of those that has been developed using open source using a 3D printer for developing its mechanical design [5].

Myoelectric is an electrical activity generated by muscle contraction. While, the manifestation of the electrical activity - called the electromyogram - is then used for prosthetic hand control [6]. Experimental techniques related to the development, recording, and analysis of myoelectric signals called Electromyography (EMG). Muscle has a function as a skeleton driving force and requires some muscles interaction, either synergistically or antagonistically. Artificial Neural Network (ANN) had been used to find out which EMG signal extraction method is optimum to recognize hand movements. The study used normal subjects as well as amputated subjects to obtain data by using six channel surface EMG (sEMG) to recognize five hand movements, namely relaxation, grasping, pinch, widening / spreading, and also pointing [7]. This method is also used to classify seven hand movements taken from four SEMG channels using ANN [8] and ten different hand movements that utilize two sEMG channels [9]. Based on these three studies, it can be concluded that sEMG and ANN can be used to predict movement generated by muscle electrical activity that is tapped using sEMG.

Based on these background, the present study designed prosthesis of bionic hand that recognize gripping, clamping, and opening palms as the basic function of the arm. This uses the myoelectric signal information generated by subject by using three sEMG channels. Three myoelectric signals will be tapped by sEMG and processed analogously through a series of electronic components. The result of this signal processing were used as input for ADC of microcontroller, Arduino Due based on ARM Cortex M3+ microcontroller. 
Thus it has good computation ability. The Arduino Due microcontroller was given an embedded program that contains ANN and also extraction feature which recognizes the myoelectric signal pattern as input for microcontroller. It was programmed to control the DC motor from the design of hand prostheses. By controlling DC motion, it was expected that the design of arm prosthesis could simulate the movement of grasping, pinning, and opening the palm of the hand by the movement of the subject.

\section{MATERIAL AND METHOD}

\section{A. Myoelectric Signal Acquisition System}

The sEMG device was used to acquire the myoelectric signal from the tapped muscles, consisting of several parts in sequence i.e. instrument amplifier, active high-pass filter, active rectifier, and active notch filter. The processed myoelectric signal was then read by Arduino DUE microcontroller that uses ARM Cortex M3+ processor so that its capability for digital signal processing is fast enough [10]. The sEMG circuit electronics of the sEMG device can be seen in Fig. 1.

The instrument amplifier serves to amplify the difference of inverting and non-inverting input signals by being implemented in the form of a single IC chip. In this study, an AD620 IC was used that requires an external resistor (Rk) to adjust the gain in the range $1-10000$ times. $\mathrm{K}$ denotes the amplification of AD620 with the reinforcement value corresponding to (1). For the value of $\mathrm{Rk}=5 \mathrm{k} \Omega$, then the obtained amplification value of instrument amplifier $\mathrm{K}=$ 9.92. After testing the instrument of amplifier AD620, it was found that the experimental amplification value of $\mathrm{K}=9.88$, thus the test result of electronic circuit instrument amplifier with the result of theoretical calculation was close to the same value.

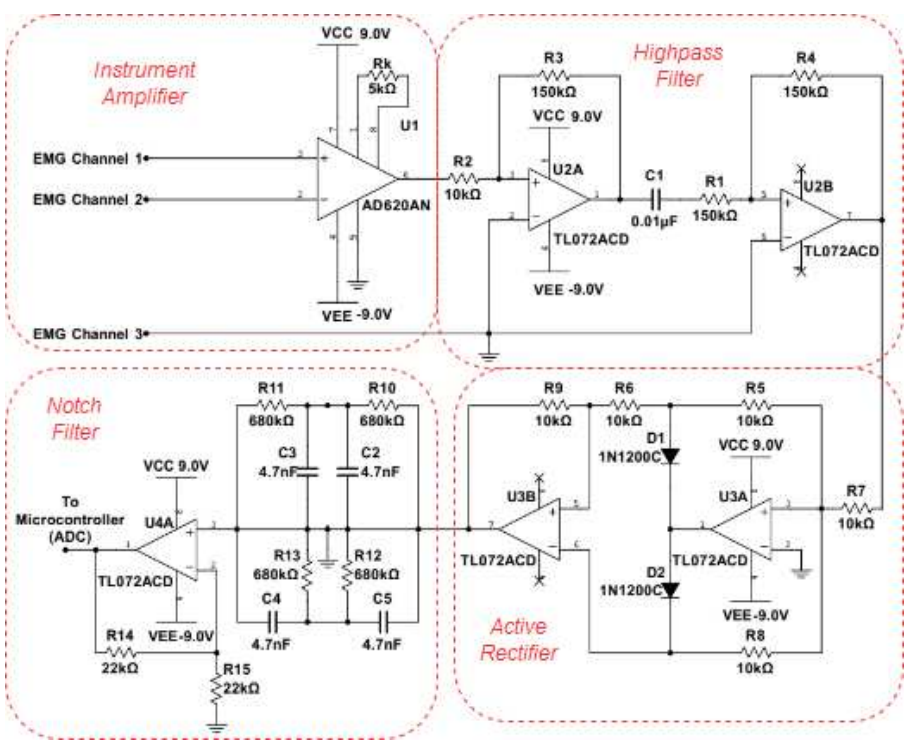

Fig. 1 sEMG electronics circuit .

$$
K=\frac{49.6 k \Omega}{R_{k}}-1
$$

The output of the instrumentation amplifier circuit would be the input of the Active High Pass filter circuit. By utilizing the TL072 IC which has two op-amps in it, it will be able to form active high-pass filter circuit (see Fig. 1). Theoretically the highpass filter (HPF) circuit has a transfer function as in (2). It can be seen that with values of R4 $=150$ $\mathrm{k} \Omega$ and $\mathrm{C} 1=0.01 \mu \mathrm{F}$ then the HPF circuit has a cut-off frequency at $\mathrm{fc}=106.2 \mathrm{~Hz}$ and with values of R3 $=150 \mathrm{k} \Omega$ and $\mathrm{R} 2=150 \mathrm{k} \Omega$ then the HPF circuit also has 15 times gain. The cutoff (fc) value was chosen with the assumption that the signals considered as noise signals with frequencies below $106.2 \mathrm{~Hz}$. This circuit was examined with the result in the form of bode diagram as the frequency response of the circuit. In Fig. 2, it can be seen that the cut-off frequency value at gain of $22 \mathrm{~dB}$ was $\mathrm{fc}=106 \mathrm{~Hz}$, therefore, the active high-pass filter circuit works well as the value of $\mathrm{fc}$ approaches the theoretical value of $106.2 \mathrm{~Hz}$.

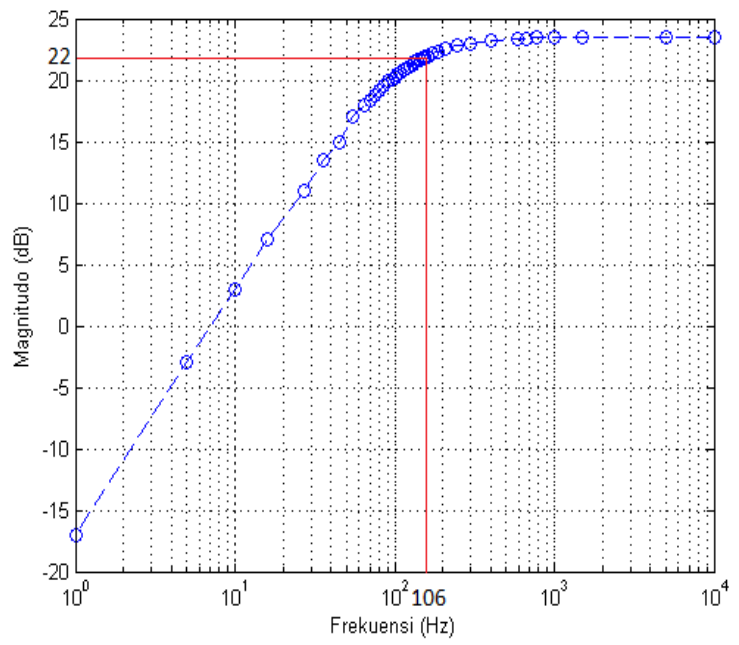

Fig. 2 Bode Diagram of Active Highpass Filter

$$
\frac{V_{\text {out }}(j \omega)}{V_{\text {in }}(j \omega)}=15 \frac{j \omega 0.015}{j \omega 0.015+1}
$$

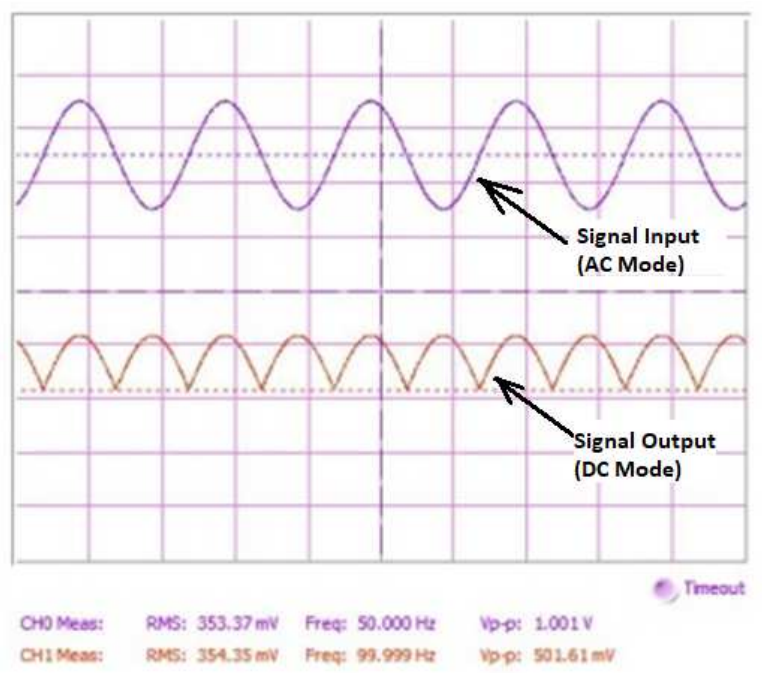

Fig. 3 Comparison between input and output signal of active circuit

The rectifier is a circuit that changes AC signals that have positive and negative polarity into DC signals that have positive polarity. The addition of this circuit was to read of 
myoelectric signals; when there is a contraction of muscle, then the myoelectric signal would be a positive value. The mechanism on how the rectifier circuit works is when the input signal has positive polarity, then the diode D1 will be on, and diode D2 will be off. Thus the rectifier circuit becomes two inverting amplifiers with the total gain of one time. Therefore, the output signal is equal to the input signal in terms of either its amplitude or polarity. While the input signal has a negative polarity the diode D1 will be off, and diode D2 will be on (see Fig. 1). Thus the rectifier circuit becomes an inverting and a non-inverting amplifier circuit so that the total gain of this circuit is -1 times. The output signal has the same amplitude as the input signal, but the polarity would be positive. From this, it was found that all output signals have positive polarity so that the output signal is a DC signal. The active rectifier circuit was tested by inputting the AC signal and see if the output signal becomes a DC signal. Test results can be seen in Fig. 3 with the output signal in the DC signal mode, thus, the active rectifier circuit was working correctly.

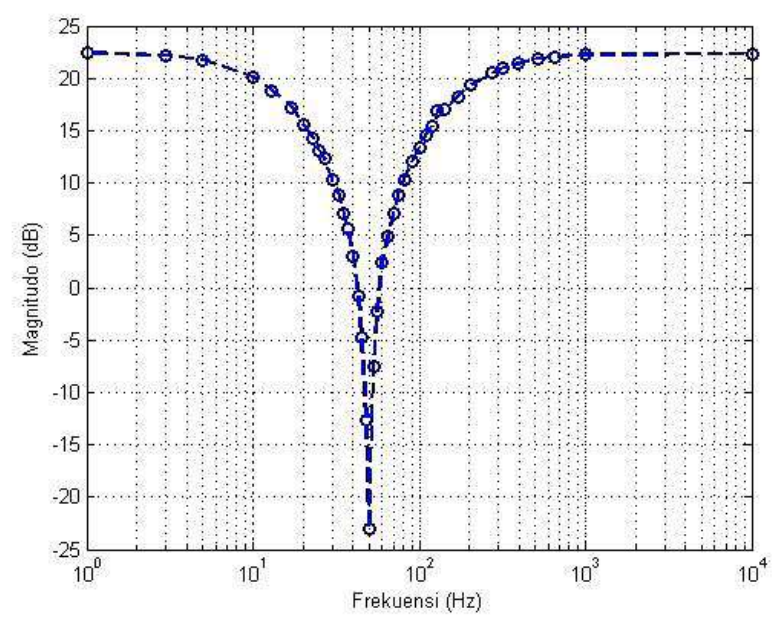

Fig. 4 Bode Diagram of Notch Filter

Notch Filter is one type of band-stop filter or band-reject filter. Band-reject filter was used to block signals with a certain frequency range. When the band stop is narrow enough, the frequency response formed is called the notch response. Notch filter has the purpose of eliminating interference due to power line noise at a frequency of $50 \mathrm{~Hz}$. It can be seen that the notch filter circuit has a non-inverting amplifier section. The circuit has a transfer function as in (3). From (3) it can be seen that the middle-frequency value $\left(f_{o}\right)$ of the filter is $49.8 \mathrm{~Hz}$. After this circuit was made, it was tested in the same way as those to the active high-pass filter by observing the Bode Diagram (Fig. 4). It can be seen that for is at $49.8 \mathrm{~Hz}$, which is the frequency value that needs to be removed due to power line noise.

$$
\frac{V_{\text {out }}(j \omega)}{V_{\text {in }}(j \omega)}=\frac{13.22\left(1-(\omega 0.003196)^{2}\right)}{\left(1-(\omega 0.003196)^{2}\right)+j \omega 0.003196}
$$

\section{B. Myoelectric Signal Processing}

Movements that was being implemented on the prosthetic hand is the movements of grasping, open grip and pinch. Physiologically these three movements involve two muscles, namely the extensor digitorum and the superficial flexor digitorum. In order to distinguish the three prosthetic hand movements, the myoelectric signal was intercepted by a three-channel sEMG device with one channel each on the extensor digitorum, the upper and lower flexor digitorum superficial as shown in Fig. 5.

After the myoelectric signals from each muscle have been obtained, extraction feature of the myoelectric signal was performed to get the conclusion of arm movements; grasping, opening grip or pinch that was implemented on the prosthetic hand. The methods of extraction feature used were those that have been developed in [7],[11] and [12] in which both have developed 21 features of the myoelectric signal: $x[\mathrm{n}]=\left\{x_{1}[\mathrm{n}], x_{2}[\mathrm{n}], \ldots ., x_{2 l}[\mathrm{n}]\right\}$, to differentiate arm movements.

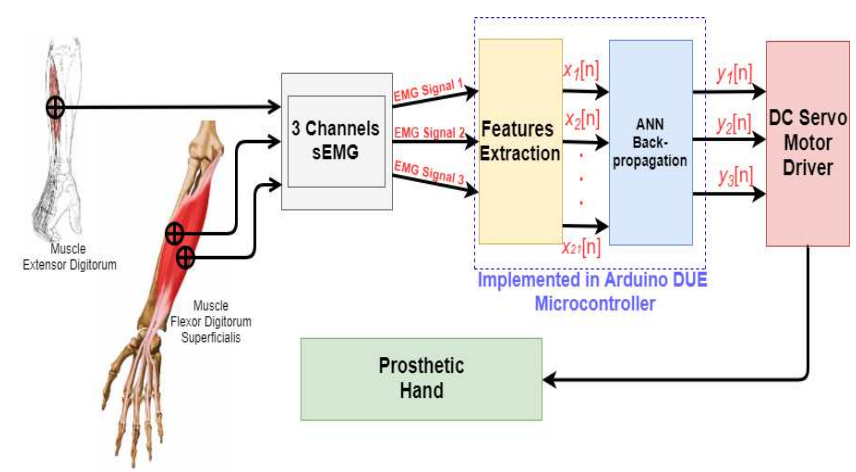

Fig. 5 Comparation between input and output signal of active circuit

Features that have been obtained were then used for arm motion classification using artificial neural network (ANN) Backpropagation, where the output is the result of whether the arm is gripping, opening the grip or pinch, symbolized by $y[n]=\left\{y_{1}[\mathrm{n}], y_{2}[\mathrm{n}], y_{3}[\mathrm{n}]\right\}$. The process of feature extraction and ANN Backpropagation was implemented on the Arduino DUE microcontroller, which then actuates the prosthetic hand movement using DC Servo Motor.

\section{ANN Backpropagation Architecture}

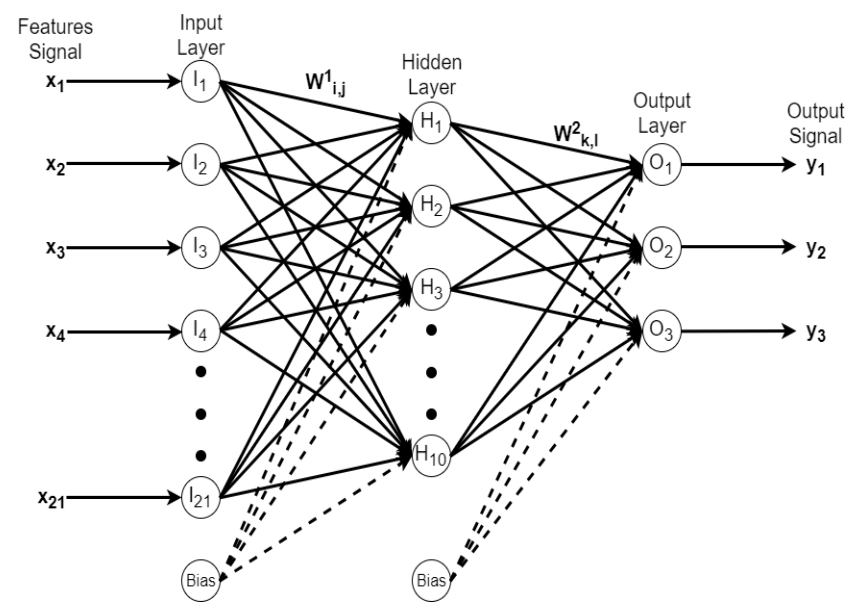

Fig. 6 ANN Backpropagation Architecture 
As shown in Fig. 5, ANN architecture consists of three layers; the input layer, hidden layer, and output layer. The number of neurons in hidden layer is 10 neurons [7] [8] [11]. The number of neurons in the input layer is 21 neurons, in which three EMG data has seven extraction features in each of them. The output layer has 3 neurons. The output layer will have matrix of target values for each type of movements as described in Table 1. The activation function of the hidden layer was using the tangent sigmoid formulated with (4) and for the activation function of output layer was using pure linear formulated by equation (5) with $H_{l}$ is activation function on the hidden layer and $O_{m}$ is the activation function at the output layer. The learning method used in ANN has a goal to get the optimum weight and bias values, which can be used for the implementation of embedded ANN. Backpropagation algorithm was being used for ANN learning, type of feed forward. If there is still an error in ANN learning then the weighting factor on ANN architecture would be updated by using (6) with $W_{j i}[\mathrm{n}+1]$ is updated weighting factor, $\mathrm{W}_{\mathrm{ji}}[\mathrm{n}]$ is previous weighting factor, $\mu$ is learning parameter and $\delta_{\mathrm{j}}$ is local error value on ANN learning.

$$
\begin{gathered}
H_{l}=\frac{2}{1+e^{\left(-2 I_{i}\right)}}-1 \\
O_{m}=y_{k} \\
W_{j i}[n+1]=W_{j i}[n]+\mu \delta_{j} y_{k}
\end{gathered}
$$

TABLE I

TARGET MATRIX OF PROSTHETIC HAND MOTION

\begin{tabular}{|c|c|c|c|}
\hline \multirow{4}{*}{ Target } & \multicolumn{3}{|c|}{ Target Motion } \\
\cline { 2 - 4 } Matrix & Grasping & Open Grip & Pinch \\
\cline { 2 - 4 } & 1 & 0 & 0 \\
\cline { 2 - 4 } & 0 & 1 & 0 \\
\cline { 2 - 4 } & 0 & 0 & 1 \\
\hline
\end{tabular}

\section{Features Extraction of Myoelectric Signal}

The hand gestures (grasp, pinch, and open grasp) is recognized by myoelectric signal classification. The most important thing in recognizing the myoelectric signal pattern depends on the selection of features that represent the raw myoelectric signal for the classification itself.

\begin{tabular}{|c|c|c|}
\hline No. & Features Extraction method 1 [7] & Features Extraction method 2 [11] \\
\hline 1 & $\begin{array}{l}\text { Mean (M) } \\
M=\frac{1}{N} \sum_{i=1}^{N} x_{i}\end{array}$ & $\begin{array}{l}\text { Mean Absolute Value (MAV) } \\
M A V=\frac{1}{N} \sum_{i=1}^{N}\left|x_{i}\right|\end{array}$ \\
\hline 2 & Root Mean Square $(\mathrm{RMS}) \mathrm{RMS}=\sqrt{\frac{1}{N} \sum_{i=1}^{N} x_{i}^{2}}$ & $\begin{array}{l}\text { Root Mean Square (RMS) } \\
R M S=\sqrt{\frac{1}{N} \sum_{i=1}^{N} x_{i}^{2}}\end{array}$ \\
\hline 3 & $\begin{array}{c}\text { Willison Amplitude (WA) WA }=\frac{1}{N} \sum_{i=1}^{N-1} f\left(x_{i}-x_{i+1}\right) \\
f(x)=\left\{\begin{array}{l}1, x \geq \text { treshold } \\
0, \text { otherwise }\end{array}\right.\end{array}$ & $\begin{array}{c}\text { Zero Crossing (ZC) } Z C=\sum_{i=1}^{N} \operatorname{sgn}\left(-x_{n} \times x_{n+1}\right) \\
\operatorname{sgn}(x)=\left\{\begin{array}{l}1, x>0 \\
0, \text { otherwisw }\end{array}\right.\end{array}$ \\
\hline 4 & $\begin{array}{l}\text { Slope Sign Change (SSC) } \\
\text { SSC }=\frac{1}{N} \sum_{i=1}^{N-1} f\left[\left(x_{i}-x_{i-1}\right)\right. \\
\left.\left(x_{i}-x_{i+1}\right)\right] \\
f(x)=\left\{\begin{array}{l}1, x \geq \text { treshold } \\
0, \text { otherwise }\end{array}\right.\end{array}$ & $\begin{array}{l}\text { Slope Sign Change (SSC) } \\
S S C=\frac{1}{N} \sum_{i=1}^{N-1} f\left[\left(x_{i}-x_{i-1}\right)\right. \\
\left.\left(x_{i}-x_{i+1}\right)\right] \\
f(x)=\left\{\begin{array}{l}1, x \geq \text { treshold } \\
0, \text { otherwise }\end{array}\right.\end{array}$ \\
\hline 5 & $\begin{array}{l}\text { Simple Square Integral (SSI) } \\
S S I=\sum_{i=1}^{N} x_{i}^{2}\end{array}$ & $\begin{array}{l}\text { Standart Deviation }(\text { SD) } \\
S D=\sqrt{\frac{1}{N-1} \sum_{i=1}^{N}\left(x_{n}-\bar{x}\right)^{2}}\end{array}$ \\
\hline 6 & $\begin{array}{l}\text { Variance }(\text { V) } \\
V=\frac{1}{N-1} \sum_{i=0}^{N}\left(x_{i}-M\right)^{2}\end{array}$ & $\begin{array}{l}\text { Variance of EMG }(\text { VAR }) \\
V A R=\frac{1}{N-1} \sum_{i=1}^{N} x_{i}^{2}\end{array}$ \\
\hline 7 & $\begin{array}{l}\text { Waveform Length (WL) } \\
W L=\sum_{i=1}^{N-1}\left|x_{i+1}-x_{i}\right|\end{array}$ & $\begin{array}{l}\text { Waveform Length (WL) } \\
W L=\sum_{i=1}^{N-1}\left|x_{i+1}-x_{i}\right|\end{array}$ \\
\hline
\end{tabular}

TABLE II

FEATURES EXTRACTION METHOD

Therefore, in this paper, two different sets of feature extractions were used as previously mentioned [7] referred to methods 1 and [11] which then called method 2. Both sets of extraction feature were compared for its application to
ANN. Both sets of extraction feature can be seen in Table 2, where $N$ is the length of the sampled myoelectric signal of each channel, and $i$ is sampled instance. These two sets of extraction features were tested for higher accuracy when 
classifying motion by ANN. Some types of features used in [7] and [11] are closely related to the physiological state of the muscle. Root Mean Square is associated with an indication of muscle contraction by a constant force before experiencing muscle fatigue. Willison Amplitude is an indication of Motor Unit Action Potential. Simple Square Integral is an indication of myoelectric signal energy and Variance which are related to signal power.

\section{E. Prosthetic Hand Design}

TACT Hand is a mechanical design of Prosthetic Hand and myoelectric [5]. The prototype design of a hand prosthetic as an actuator is the result of an open source 3D print as it can be seen in Fig. 7. The design of the TACT has a physical appearance similar to the anatomy of the human hand. All resources on TACT Hand are freely available on the page of Patrick Slade as the developer of TACT Hand, so that from this source, it can be printed directly on a 3D printing machine.

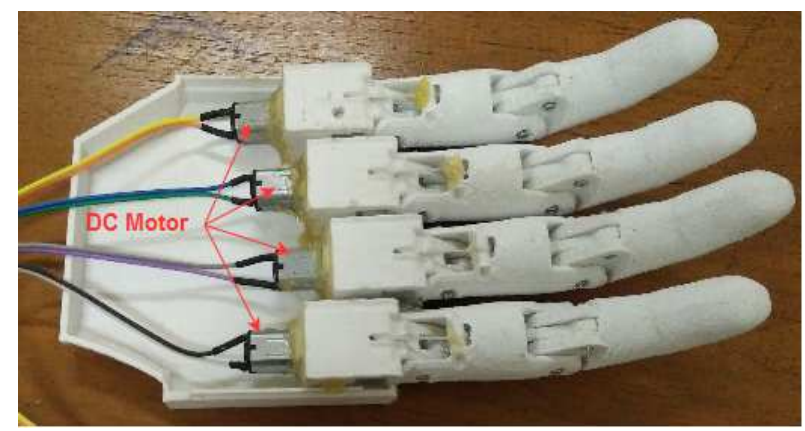

Fig. 7 3D Printed Prosthetic Hand

The control of this mechanic hand-prosthesis was using an embedded DC motor for each TACT mechanical finger. DC motor can reverse the direction of rotation by reversing the polarity of DC motor input. This polarity reversal control uses the H-Bridge circuit. In general, the H-Bridge circuit uses transistors that act as a switch. Therefore this switch function can be replaced with IC L293D IC.

\section{RESULT AND DISCUSSION}

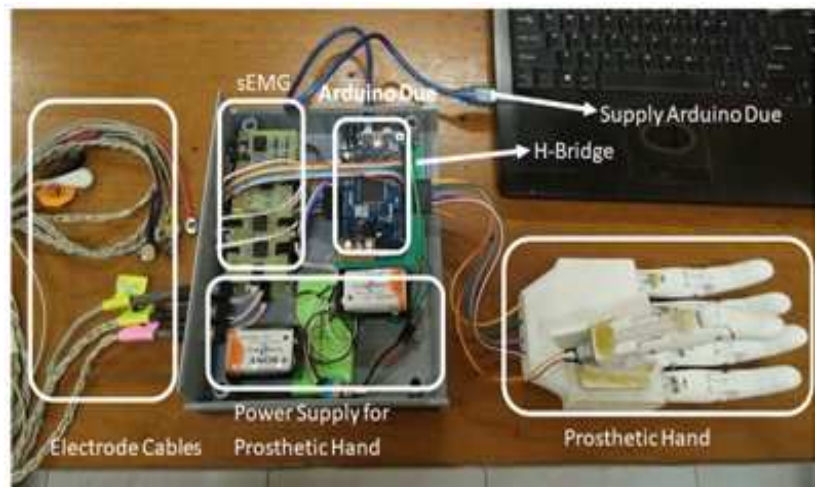

Fig. 8 Prosthetic Hand Hardware System

Fig. 8 shows the implementation of the electronic circuit sEMG, Arduino DUE microcontroller, and prosthetic hand that has been made.

\section{A. Myoelectric Feature Extraction Examination}

Five subjects were selected with age in the range of 19-22 year old. Each subject was requested to perform grip movements, opening the grip and pinch. Each subject produces 13 sets of myoelectric signal data; 10 data sets were used as training data and 3 sets as test data. Thus, the total ANN Backpropagation algorithm would be given 50 sets of training data and 15 sets of test data with 2 types of extraction features. Each method of extraction feature has 7 features and the myoelectric signal was acquired from 3 muscles. Therefore, the input from ANN Backpropagation algorithm was 21 features.

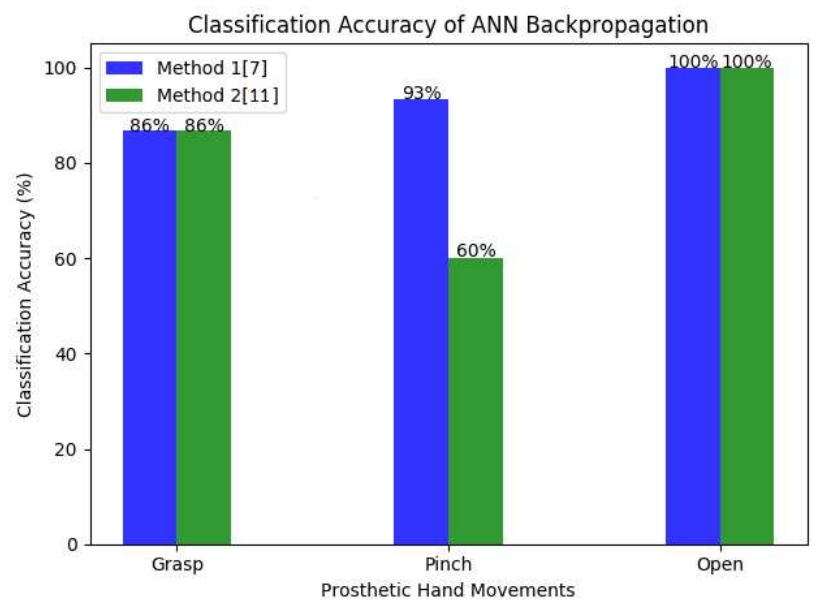

Fig. 9. Accuracy of Classification using ANN Backpropagation between Features Extraction method 1 and 2

Training with 50 sets of data as previously described was carried out, and ANN Backpropagation algorithm learning outcomes were tested with 15 sets of test data. In this test, the accuracy of the classification results of the ANN Backpropagation algorithm was compared for the ability in classifying provided test data correctly. In case of the given test data is a myoelectric signal of grasping movement, then the conclusion of ANN Backpropagation algorithm should be grasping. This was considered that ANN Backpropagation algorithm could classify correctly. This was applied similarly for grip and pinch movements. Experimental results can be seen in Fig. 8. Extraction feature method 1 showed an $86 \%$ accuracy for grasping, 93\% for pinching and $100 \%$ for gripping. Extraction feature method 2 showed $86 \%$ accuracy for grasping, $60 \%$ for pinching, and $100 \%$ for gripping. Therefore, extraction features method 1 , developed by Rillo et al., showed better accuracy, and then it was used as the extraction feature method in the implementation of prosthetic hand movement control.

\section{B. Prosthetic Hand's Real-Time Control Examination}

Using feature extraction method 1 and ANN Backpropagation algorithm as a classifier of arm movements, the prosthetic hand movement was further examined for its controlling precision by the myoelectric signal of subjects muscle in real time. Four subjects then called subject 1, 2, 3, and 4 . The height-weight ratio characterized each subject. Subject $1,2,3$ and 4 have ratio values of $3.47 ; 3.17 ; 3.14$ and 2.6, respectively. This characterization is necessary to differentiate the muscle mass of each subject, subject with larger muscle is relatively more detectable for the 
installation of electrodes as well as the power of the myoelectric signal. This examination process is shown in Fig. 10 .

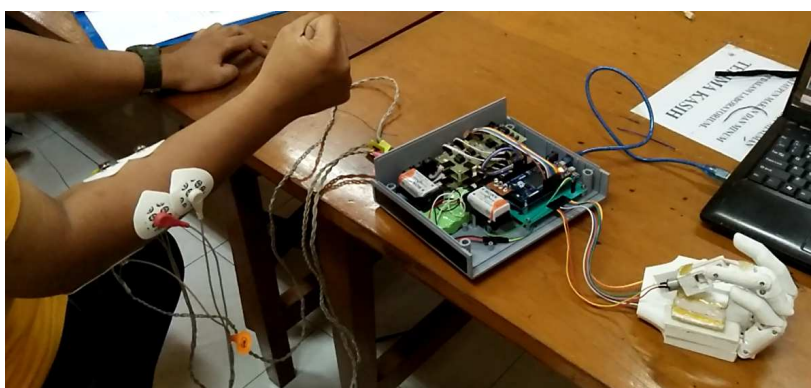

(a)

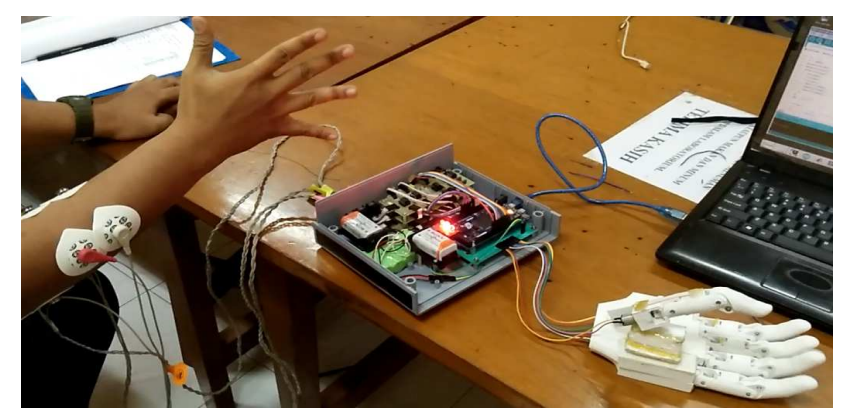

(b)

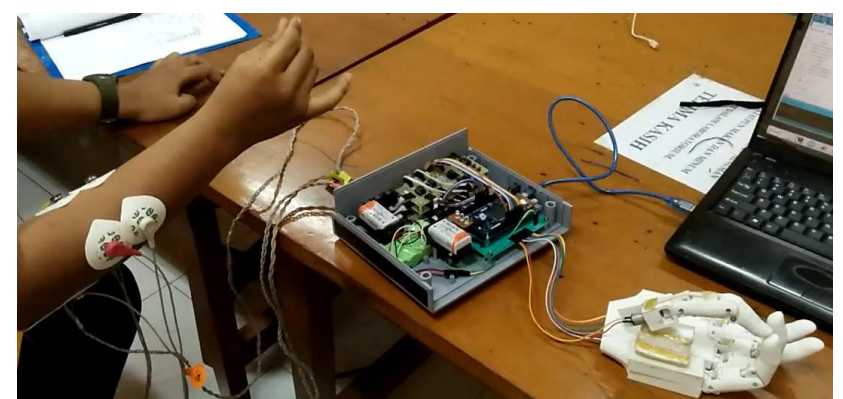

(c)

Fig. 10 Prosthetic Hand Control Examination (a) Grasping (b) Open Hand (c) Pinch

Furthermore, the four subjects were required to perform gripping movements, open grips, and pinch randomly; a movement that is performed sequentially and not freely based on their own, making the same movement in sequence. A total number of attempted movements were the same for all subjects, i.e., 25 times. Afterward, the motion accuracy (percent), i.e., number of successfully mimicking the subject movement was calculated. The results of the first experiment can be seen in Fig. 10. The success rate of prosthetic hand control was $85.71 \% ; 84.62 \% ; 83.87 \%$ and $76.92 \%$ for subjects 1, 2, 3, and 4, respectively. Moreover, further improvement of accuracy was observed by utilizing the test data of the first experiment into training data for ANN Backpropagation and recalculated for the value of accuracy, as shown in Fig. 11.

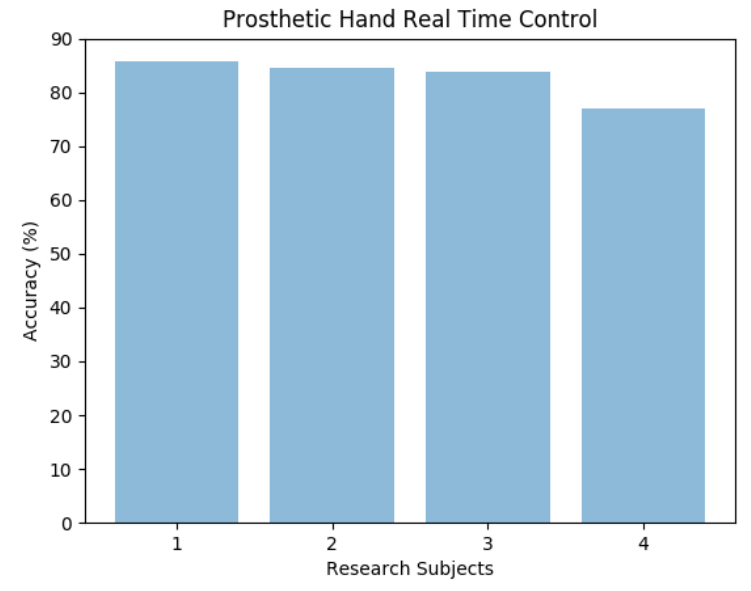

Fig. 11 Accuracy of Prosthetic Hand Gesture (First Attempt)

In Fig. 12, it can be seen that there was an increase in accuracy to $90.32 \% ; 90 \% ; 87.1 \%$ and $87.1 \%$ for subjects 1 , 2,3 and 4, respectively. Thus it can be concluded that the more rigorous the training data being input to ANN Backpropagation on control of the prosthetic hand, its accuracy was increased. In addition, higher accuracy was produced from subjects who have a high body weight/height ratio with a large muscle. Therefore, the accuracy of Prosthetic Hand control by using myoelectric signal depends on the muscle size of subjects.

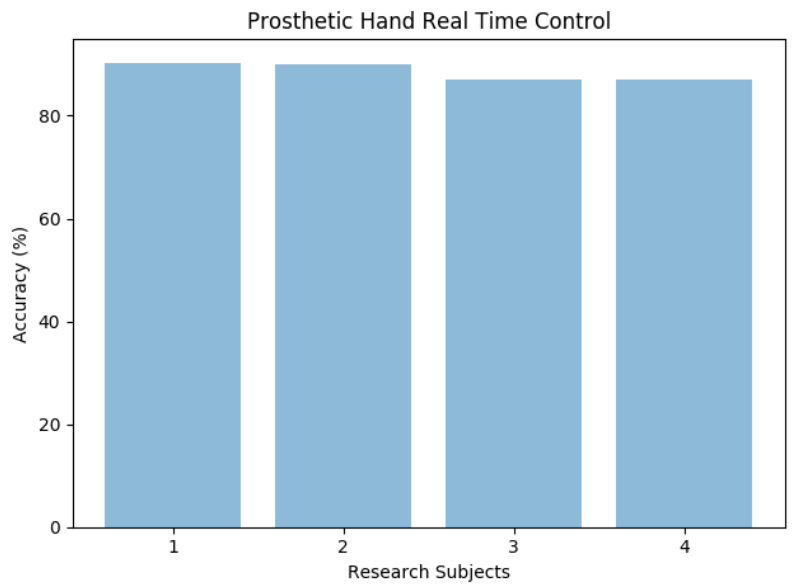

Fig. 12 Accuracy of Prosthetic Hand Gesture (Second Attempt)

\section{CONCLUSIONS}

The prosthetic hand that controlled by a myoelectric signal from flexor digitorum and extensor digitorum muscles had been designed and implemented successfully. It used three channels sEMG that one channel for flexor digitorum and two channels for extensor digitorum. Three hand gestures (grasp, open grasp, and pinch) are imitated by the prosthetic hand. The accuracy of this system were $85.71 \%$; $84.62 \% ; 83.87 \%$ and $76.92 \%$ for subjects $1,2,3$ and 4 who have height-weight ratio values of $3.47 ; 3.17 ; 3.14$ and 2.6 , respectively. 


\section{ACKNOWLEDGMENT}

We would like to thank Faculty of Science and Technology, Universitas Airlangga Surabaya for research grant via Young Lecturer Research Grant 2017 number : SP POPA 628/UN3.6/KU/2017.

\section{REFERENCES}

[1] K. Lamb. (2016), Amputation. [Online]. Available: https://vascular.org/patient-resources/vascular-treatments/amputation.

[2] Ottobock. (2016), Rehabilitation. [Online]. Available: http://www.ottobock.id/id/prosthetics/information-foramputees/from-amputation-to-rehabilitation/rehabilitation/.

[3] R. LeMoyne, Advance for Prosthetic Technology: From Historical Perispective to Current Status to Future Application, Tokyo, Springer, 2016.

[4] L. McLelan and R. N. Scott, Powered Upper Limb Prostheses: Control, Implementation and Clinnical Application, New York, Springer, 2016.

[5] P. Slade, A. Akhtar, M. Nguyen and T. Bretl, "Tact: Design and performance of an open-source, affordable, myoelectric prosthetic hand," 2015 IEEE International Conference on Robotics and Automation (ICRA), Seattle, WA, 2015, pp. 6451-6456.

[6] P. Geethanjali and K. K. Ray, "A Low-Cost Real-Time Research Platform for EMG Pattern Recognition-Based Prosthetic Hand," in IEEE/ASME Transactions on Mechatronics, vol. 20, no. 4, pp. 19481955, Aug. 2015
[7] F. Riillo, L.R. Quitadamo, F. Cavrini, E. Gruppioni, C.A. Pinto, N. Cosimo Pastò, L. Sbernini, L. Albero and G. Saggio, "Optimization of EMG-based hand gesture recognition: Supervised vs. unsupervised data preprocessing on healthy subjects and transradial amputees",in Biomedical Signal Processing and Control, Vol. 14, pp $117-125,2014$

[8] U. Baspinar, H. Selcuk Varol, and V. Y. Senyurek, " Performance Comparison of Artificial Neural Network and Gaussian Mixture Model in Classifying Hand Motions by Using sEMG Signals ", in Biocybernetics and Biomedical Engineering, Vol. 33, Issue 1, pp 3345, 2013.

[9] R. N. Khushaba, S. Kodagoda, M. Takruri, and G. Dissanayake, " Toward improved control of prosthetic fingers using surface electromyogram (EMG) signals ", in Expert Systems with Applications, Vol. 39, Issue 12, pp. 10731-10738, 2012.

[10] L. Bitjoka, M. Ndje, A.T. Boum, J.S. Manguele," Implementation of Quadratic Dynamic Matrix Control on Arduino DUE ARM Cortex M3+ Microcontroller Board ", in Journal of Engineering and Technology, Vol. 6, pp. 682 - 695, 2017

[11] M. R. Ahsan, M. I. Ibrahimy and O. O. Khalifa, "Electromygraphy (EMG) signal based hand gesture recognition using artificial neural network (ANN)," 2011 4th International Conference on Mechatronics (ICOM), Kuala Lumpur, 2011, pp. 1-6.

[12] M. N. Mohd Nor,R. Jailani,N. M. Tahir,Ihsan Mohd Yassin,Zairi Ismael Rizman and Rahmat Hidayat,"EMG Signals Analysis of BF and RF Muscles In Autism Spectrum Disorder (ASD) During Walking," International Journal on Advanced Science, Engineering and Information Technology, vol. 6, no. 5, pp. 793-798, 2016. [Online]. Available: http://dx.doi.org/10.18517/ijaseit.6.5.1205. 